\title{
Experimental Verification of Inertial Navigation with MEMS for Forensic Investigation of Vehicle Collision
}

\author{
Srdjan TADIC, Milan B. VUKAJLOVIC \\ School of Electrical Engineering, University of Belgrade, Bulevar kralja Aleksandra 73, 11120 Belgrade, Serbia \\ srdjan.tadic@gmail.com,milanvukajlovic86@gmail.com
}

Manuscript received October 30, 2015

\begin{abstract}
This paper studies whether low-grade inertial sensors can be adequate source of data for the accident characterization and the estimation of vehicle trajectory near crash. The paper presents outcomes of an experiment carried out in accredited safety performance assessment facility in which full-size passenger car was crashed and the recordings of different types of motion sensors were compared to investigate practical level of accuracy of consumer grade sensors versus reference equipment and cameras. Inertial navigation system was developed by combining motion sensors of different dynamic ranges to acquire and process vehicle crash data. Vehicle position was reconstructed in three-dimensional space using strapdown inertial mechanization. Difference between the computed trajectory and the ground-truth position acquired by cameras was on decimeter level within short time window of $750 \mathrm{~ms}$. Experiment findings suggest that inertial sensors of this grade, despite significant stochastic variations and imperfections, can be valuable for estimation of velocity vector change, crash severity, direction of impact force, and for estimation of vehicle trajectory in crash proximity.
\end{abstract}

\section{Keywords}

Accelerometer, gyroscopes, vehicle crash testing, navigation, positioning

\section{Introduction}

Modern vehicles are equipped with clusters of inertial sensors as parts of safety and stability mechanisms. Crash Event Data Recorders (EDRs), which are today standard equipment of passenger cars, also provide sensor and vehicle instrument captures for post-accident forensic analysis [1]. Another source of collision data are after-market insurance-telematics devices [2] as they estimate severity of impact events and direction of the force. Still, in many cases, fatal vehicle crashes become a matter of dispute in forensic investigation. Existing crash analysis tools [3] provide only directions of approach to investigators, and therefore can lead to ambiguous conclusions in otherwise solvable cases. The potential of different motion sensors already deployed in vehicles strongly overcomes their current use as autonomous sources of crash information. These are mostly low performance "consumer-grade" sensors considering bias-instabilities, noise density and angular random-walk. It is valuable to study possibility to incorporate this class of sensors as parts of strap-down inertial navigation system (SDINS, [4]) that would be used for short-term vehicle trajectory estimation in crash. SDINS combines accelerometers and rate gyro outputs to provide autonomous and precise navigation of an object in space. Such reduced model SDINS could become tool for objective analysis of vehicle motion and elucidation of accident causes. When possible, this approach should be integrated with the Global Navigation Satellite System (GNSS) receiver and proper fusion algorithm to provide absolute positioning [5] and tracking of motion sensor error model during regular driving conditions. At crash situation, offthe-shelf GNSS receivers cannot be easily used as a direct source of data. They rely on signal-tracking mechanisms [6] that are unreliable at high-dynamic, so receiver operation is normally guaranteed at accelerations below $50 \mathrm{~m} / \mathrm{s}^{2}$ and jerks below $20 \mathrm{~m} / \mathrm{s}^{3}$.

In the field of forensic collision reconstruction, in order to analyze the mechanical components of a dynamic impact, the overall crash severity is typically assessed by quantifying the change in velocity of the vehicle or of the occupant(s). Most of the crash-test facilities rely on camera recordings and outputs of piezoelectric accelerometers. Forensic analysis usually does not consider combining inertial sensor outputs for tracking both the vehicle angular rotation and translation because accurate quantifying of angular motion is a challenge in the extreme dynamic tests with significant shock and vibration. Applied sensors need to be capable of tolerating short duration acceleration spikes or vibration. Another challenge is increased nonlinearity of sensors out of certain measurement ranges and bandwidths. Proper crash test recording thus requires specially designed components [7]. More recent work [8], [9] studies behavior of low-cost inertial micro-electro mechanical sensors (MEMS) in dynamic conditions. One of the findings is that characteristics of the rate gyroscopes are more stable than of the accelerometers. In one of the rare examples of use of SDINS within the crash scenario [10], Inertial Measurement Unit (IMU) of higher perfor- 
mance grade was mounted inside of the test-dummy during lab test. The calculated trajectory was then compared to reference camera. The reported average position error within $300 \mathrm{~ms}$ was $2.3 \mathrm{~mm}$, with maximum of $6.1 \mathrm{~mm}$.

In the next sections, first some specifics of vehicle crash environment will be presented and how they affect typical sensor errors and its dynamics. Then, the performance of SDINS with low-cost IMU will be evaluated in the experiment carried out in a certified crash-test laboratory.

\section{Crash Environment, Kinematic Equations and Error Dynamics}

At severe collisions, crash pulse magnitude can reach 50-100 g's $\left(g \approx 9.81 \mathrm{~m} / \mathrm{s}^{2}\right)$ within $50-250 \mathrm{~ms}$ [11]. For accurate detection, the sensor sampling rates should be above $100 \mathrm{~Hz}$. Vehicle motion in crash is complex and nonlinear and it is followed by transformation of kinematic energy to structural deformation [12]. While a vehicle collides, it acts as an object with six degrees of freedom of movement in space, which means that trajectory recording with the inertial sensors requires three-axial IMU. After the recording, a common method for integration of the specific forces and angular rates which finally provides vehicle's position in space is "inertial mechanization". It is used for short-term solving of navigation differential equations in frame of interest. Mathematical foundations and SDINS, as implemented, are given in the next section.

\subsection{Tangent Frame Kinematic Equations}

Equations (1) and (2) present calculation of velocity $\left(\mathbf{V}^{N}\right)$ and position $\left(\mathbf{S}^{N}\right)$ vectors respectively [4].

$$
\begin{gathered}
\mathbf{V}^{N}=\int_{0}^{t} \mathbf{f}^{N} d t-\int_{0}^{t}\left[2 \boldsymbol{\omega}_{I E}^{N}+\boldsymbol{\omega}_{E N}^{N}\right] \times \mathbf{V}^{N} d t+\int_{0}^{t} \mathbf{g}^{N} d t, \\
\mathbf{S}^{N}=\int_{0}^{t} \mathbf{V}^{N} d t \\
\mathbf{V}^{N}=\left[\begin{array}{lll}
V_{N} & V_{E} & V_{D}
\end{array}\right]^{T}, \\
\mathbf{S}^{N}=\left[\begin{array}{lll}
S_{N} & S_{E} & S_{D}
\end{array}\right]^{T}, \\
\mathbf{f}^{N}=\left[\begin{array}{lll}
f_{N} & f_{E} & f_{D}
\end{array}\right]^{T}, \\
\mathbf{\omega}_{I E}^{N}=\left[\begin{array}{lll}
\mathbf{g}_{e} \cos \varphi & 0 & -\omega_{e} \sin \varphi
\end{array}\right]^{T}, \\
\boldsymbol{\omega}_{E N}^{N}=\left[\begin{array}{ccc}
\frac{V_{E}}{R_{p}+h} & \frac{-V_{N}}{R_{M}+h} & \frac{V_{E \tan \varphi}}{R_{p}+h}
\end{array}\right]^{T} .
\end{gathered}
$$

Velocity vector, position vector, specific forces vector and gravity vector in local NED (North-East-Down) coordinate frame are presented in (3), (4), (5) and (6) respectively. Earth rotation projections onto NED axis is shown in (7) where angular velocity of the earth rotation $\omega_{\mathrm{e}}=7.292115 \times 10^{-5} \mathrm{rad} / \mathrm{s}$, which can be neglected for short navigation with low performance sensors. Angular velocity of rotation of a navigation coordinate frame relative to the earth is shown in (8). Position coordinates longitude, latitude, height are $\lambda, \varphi, h$ respectively and $R_{M}$, $R_{p}$ are radius of curvature along Earths meridian and parallel. Time rates of position coordinates in WGS-84 (World Geodetic System, est. at 1984.) coordinate frame can be represented as in (9).

$$
\begin{gathered}
\dot{\lambda}=\frac{V_{E}}{\left(R_{p}+h\right) \cos \varphi}, \quad \dot{\varphi}=\frac{V_{N}}{R_{M}+h}, \quad \dot{h}=-V_{D}, \\
{\left[\begin{array}{llllll}
V_{N} & V_{E} & V_{D} & \lambda & \varphi & h
\end{array}\right]^{T} .}
\end{gathered}
$$

The set of navigation parameters that describe velocity vector and position of object in space can be written as in (10). This set can be calculated by numerical integration of differential equations (1), (2) and (9). Specific force vector represented in NED coordinate frame given in (3) should be derived from the acceleration measurements made in body fixed coordinate frame. Direction cosine matrix (DCM) representing the transformation between these two frames can be specified as $\mathbf{C}_{B}^{\mathrm{P}}$, pointing out by upper index $\mathrm{P}$ that the orientation of calculated platform generally differs from the actual orientation of NED frame. The orientation algorithm applied here is based on usage of quaternions in calculation of DCM and it is performed as a two-step procedure [13]. In the first step rotation of quaternion is calculated due to rotation of body relative to inertial space shown in (11). $\mathbf{Q}^{P}$ is the preliminary quaternion and $\mathbf{Q}^{F}$ is the final quaternion. Equation (12) presents rotation increment $\Delta \lambda$ due to rotation of body $\omega_{\mathrm{IB}}^{\mathrm{B}}$ measured by the set of three gyros $(p, q, r)$ rigidly fixed to the vehicle presented in (13).

$$
\begin{gathered}
\mathbf{Q}_{k+1}^{P}=\mathbf{Q}_{k}^{F} \Delta \boldsymbol{\lambda}, \\
\Delta \boldsymbol{\lambda}=\Delta \lambda_{0}+\Delta \lambda_{1} i+\Delta \lambda_{2} j+\Delta \lambda_{3} k, \\
\boldsymbol{\omega}_{I B}^{B}=\left[\begin{array}{lll}
p & q & r
\end{array}\right]^{T} .
\end{gathered}
$$

The second step presents rotation of navigation frame relative to inertial space shown in (14). Equation (15) presents quaternion increment calculated from (7) and (8).

$$
\begin{gathered}
\mathbf{Q}_{k+1}^{F}=\Delta \mathbf{m} \mathbf{Q}_{k+1}^{P}, \\
\Delta \mathbf{m}=\Delta m_{0}-\Delta m_{1} i-\Delta m_{2} j-\Delta m_{3} k .
\end{gathered}
$$

From the final quaternion shown in (18) rotation matrix $\mathbf{C}_{B}^{P}$ shown in (19) is obtained.

$$
\mathbf{Q}^{F}=q_{0}+q_{1} i+q_{2} j+q_{3} k,
$$

$$
\mathbf{C}_{B}^{P}=\left[\begin{array}{ccc}
q_{0}^{2}+q_{1}^{2}-q_{2}^{2}-q_{3}^{2} & 2\left(q_{1} q_{2}-q_{0} q_{3}\right) & 2\left(q_{1} q_{3}+q_{0} q_{2}\right) \\
2\left(q_{1} q_{2}+q_{0} q_{3}\right) & q_{0}^{2}-q_{1}^{2}+q_{2}^{2}-q_{3}^{2} & 2\left(q_{2} q_{3}+q_{0} q_{1}\right) \\
2\left(q_{1} q_{3}+q_{0} q_{2}\right) & 2\left(q_{2} q_{3}+q_{0} q_{1}\right) & q_{0}^{2}-q_{1}^{2}-q_{2}^{2}+q_{3}^{2}
\end{array}\right] .
$$


The orientation error effect consists in the existence of transformation matrix relating computed platform frame and navigation frame shown in (20), derived under the assumption that the angles $\phi_{N}, \phi_{E}, \phi_{D}$ are small.

$$
\mathbf{C}_{P}^{N}=\left[\begin{array}{ccc}
1 & -\phi_{D} & \phi_{E} \\
\phi_{D} & 1 & -\phi_{N} \\
-\phi_{E} & \phi_{N} & 1
\end{array}\right] .
$$

The resulting transformation matrix relating bodyfixed and navigation coordinate frames is shown in (21).

$$
\mathbf{C}_{\mathrm{B}}^{\mathrm{N}}=\mathbf{C}_{\mathrm{P}}^{\mathrm{N}} \mathbf{C}_{\mathrm{B}}^{\mathrm{P}} .
$$

Details of this mathematical apparatus, as implemented for this test, are given in Fig. 1 [4]. These orientation and integration algorithms have some basic simplifications from the standard scheme from textbooks - e.g. we did not consider Coriolis correction due to very limited sensor performance.

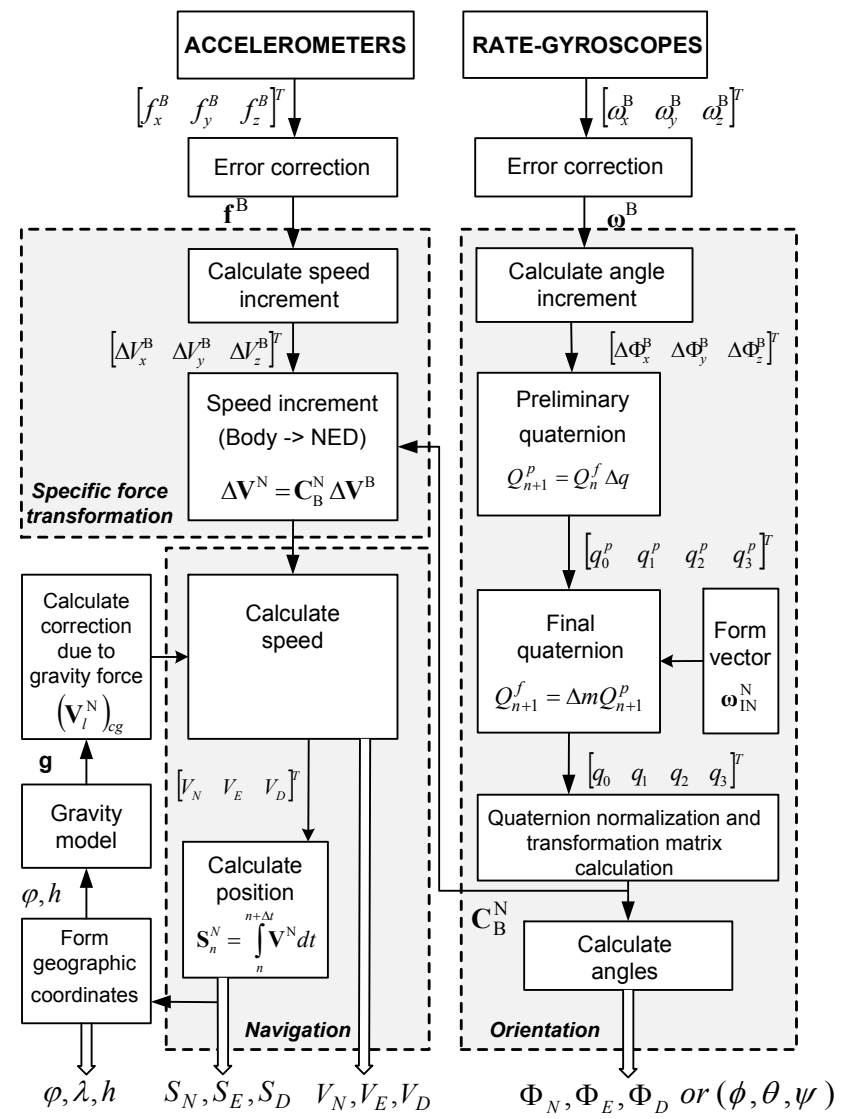

Fig. 1. SDINS processes as implemented in the embedded software (General SDINS mechanization).

\subsection{Main Sources of Error}

MEMS of this grade have extreme stochastic variances and their error characteristics change rapidly. Calculated position quickly drifts from real-world values, due to the MEMS errors [14], IMU mounting imperfections, and the influence of environment. Conventional mechanization is thus challenging for MEMS. Inertial Navigation System (INS) position error increases over time, due to gyro-drifts and sensor biases, and over traveling distance due to accelerometer scale factors and orientation misalignments. Considering practically feasible IMU mounting and initialization, the greatest influence upon the position error, for periods of up to 5-10 s and out of crash, comes from the initial speed assessment, followed by the accelerometer bias, and then the gyro bias [15]. Other effects could be neglected in similar cases. At crash conditions, nonlinearity and "g-sensitivity" are extra error sources. They are hard to model and suppress in the pre-crash period. Measurement can be further degraded by vibrations if they are not compensated by rubber dumpers.

\section{Experimentation Setup}

Test setup consisted of the lab's reference piezoelectric accelerometers (LAB1, LAB2, Fig. 2, and Tab. 1), custom test devices bolted to the vehicle chassis at two different positions on the vehicle (IMU1, IMU2) and two high-speed cameras (1000 frames/s, 1.3 megapixels). Reference sensors were compliant to SAE J211 [16] and ISO 6487 [17] as the main performance requirements of the crash test data acquisition systems. Recording of reference sensors and cameras was triggered by the same pulse so entry position may be considered as identical for all sensors. IMU1/2 combined multiple accelerometers to get high range, low noise, good resolution and stability. As system used both analog and digital sensors, a dedicated microcontroller was used for data acquisition and synchronization.

A passenger vehicle was dragged along a straight path and collided with a relatively narrow metal pole offset $0.4 \mathrm{~m}$ from its longitudinal axis (Fig. 3) resulting in a pole penetration to the car. Recording of lab sensors (LAB1/2) outputs started $100 \mathrm{~ms}$ before impact, and IMU1/2 outputs were captured from steady position of the vehicle with warm-up period of 10 minutes to avoid transients. Laboratory equipment recorded a crash-entry speed of $7.1 \mathrm{~m} / \mathrm{s}$. Accelerometers with lower dynamic range ("low-G") were used as the single available reference for the removal of biases of "high-G" sensors, neglecting the axis misalignment and their own biases which were on $0.2 \mathrm{~m} / \mathrm{s}^{2}$ level. At the worst case, bias of "high-G" accelerometer was estimated to $11.91 \mathrm{~m} / \mathrm{s}^{2}$. As sensors and camera reference points were not co-located, measurements were mathematically translated to a common reference point ("lever arm compensation") which matches a vehicle marking in central area of camera and with insignificant level of car's structural deformation. Vehicle 3D trajectory was finally reconstructed using SDINS built upon the four sensor platforms (Fig. 4). The only available rate gyros in test were those from IMU1/2.

The high-speed cameras provided positional data based on black-yellow markers spread over the vehicle. In 


\begin{tabular}{|l|l|c|c|}
\hline Sensor & Part & $\begin{array}{l}\text { Output } \\
\text { data rate }\end{array}$ & $\begin{array}{l}\text { Dynamic } \\
\text { Range }\end{array}$ \\
\hline LAB & ENDEVCO 7264B-500 & $10 \mathrm{kHz}$ & $\pm 500 \mathrm{~g}$ \\
\hline IMU low-G & LIS302DLH & $200 \mathrm{~Hz}$ & $\pm 2 \mathrm{~g}$ \\
\hline IMU high-G & MMA3202, MMA1213 & $200 \mathrm{~Hz}$ & $\pm 100 \mathrm{~g}$ \\
\hline IMU gyro & IDG-500, ISZ-500 & $200 \mathrm{~Hz}$ & $\pm 500^{\circ} \mathrm{s}$ \\
\hline
\end{tabular}

Tab. 1. List of sensors with basic characteristics.

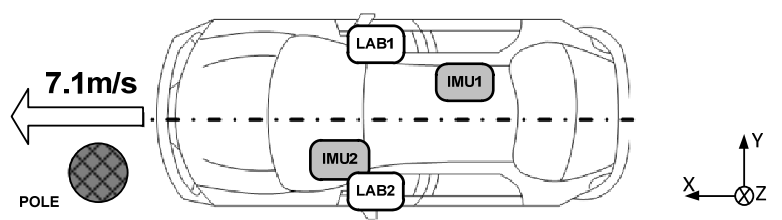

Fig. 2. Sensors positions.

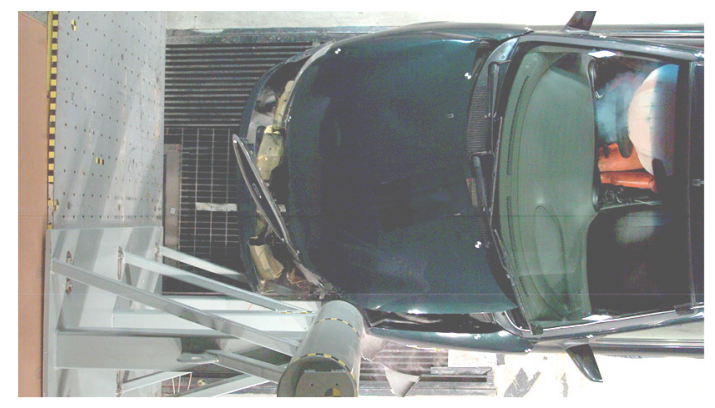

Fig. 3. Test-crash as recorded by a high-speed camera.

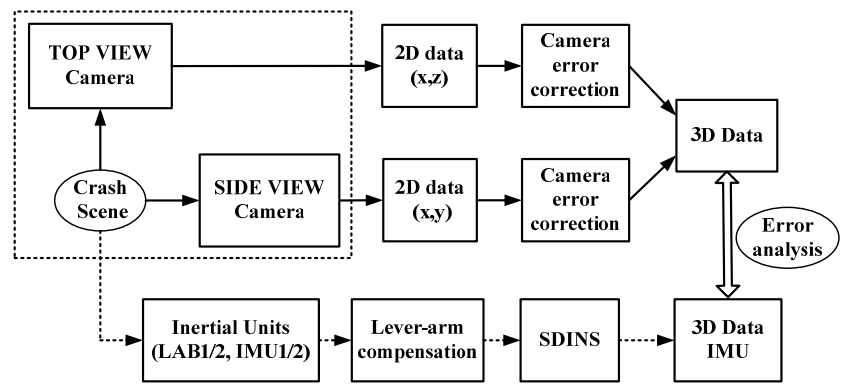

Fig. 4. Cameras and sensors readings processing.

general, accuracy of the camera readings depends on the recorded scene but also on the optical, geometrical and digital characteristics of the camera. After compensating known camera errors (such as errors caused by imageframe transformation) and minimizing lens-distortion effects by tracking only those markings which were close to the center of the scene, top and side view camera readings were combined into a "ground-truth" reference 3D trajectory (Fig. 5).

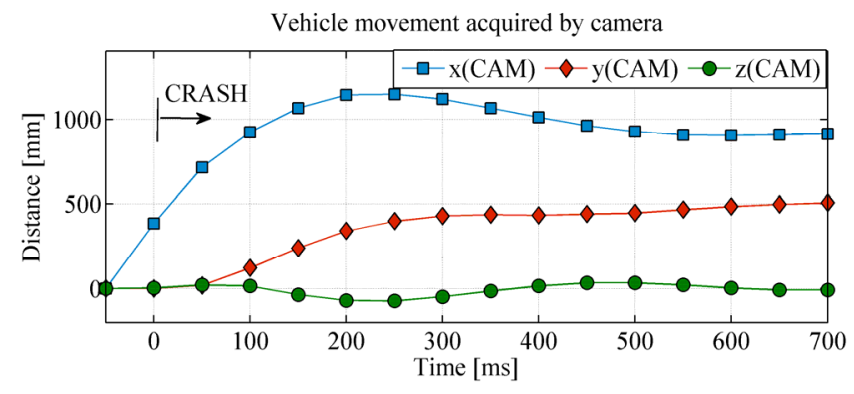

Fig. 5. Vehicle "crash trajectory" as recorded by camera.

\section{Results}

Low cost sensors and reference equipment were compared in three aspects: 1) as raw data, 2) estimation of crash severity and direction of impact force, and 3) determining vehicle trajectory against "ground-truth" acquired by cameras.

Figure 6 compares in time-domain measurements acquired by low- and high-range accelerometers from IMU1/2 with the corresponding LAB1/2 sensors. Only data along longitudinal axis were presented as due to highest impact forces they clearly show similarities, but also differences between recordings originating from various sensors. Reference lab data were down-sampled to $200 \mathrm{~Hz}$. Results are shown from both - the left and the right-side mounted groups of sensors. As seen, the "low-G" sensors (on both units) were mostly saturated during crash. Still, they play a very significant role in coarse calibration of "high-G" components as bias is usually proportional to measurement dynamic range.

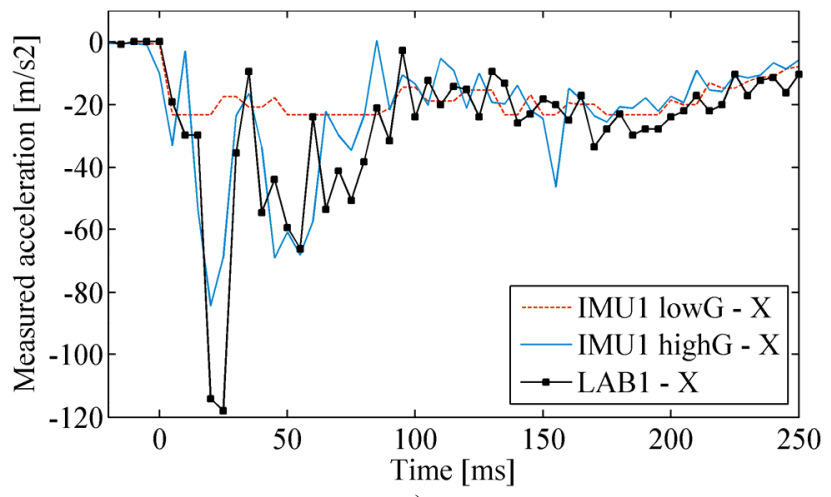

a)

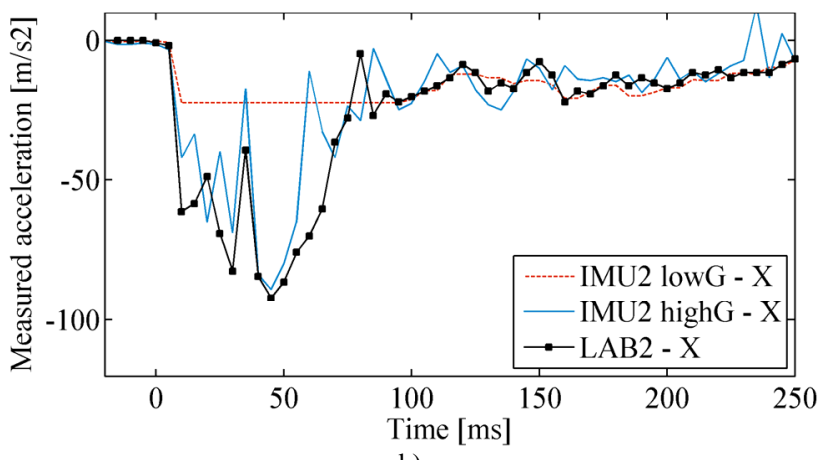

b)

Fig. 6. Longitudinal acceleration a) left and b) right-side mounted.

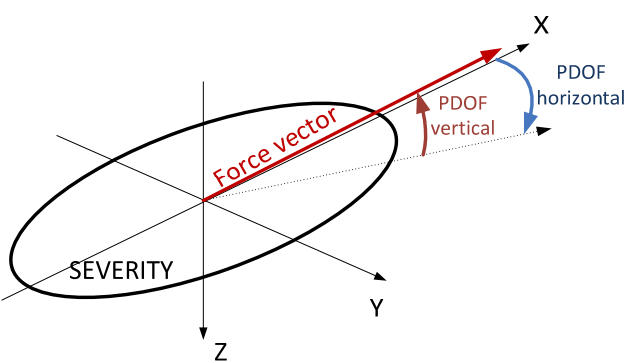

Fig. 7. Principal Direction of Force (PDOF). 
For nowadays telematics services which are relaying on near real-time access to driving data, it is desired that in case of a traffic accident, in-vehicle EDRs are able to estimate crash severity or at least with certain accuracy to distinguish type of crash. It is very important feature of EDRs as it may be further used as input for estimating passenger injury severity and improving emergency and post-injury services. Crash severity is usually estimated according to the change of the speed vector ("delta-V") as measured over the short time-window (herein $30 \mathrm{~ms}$ ). Crash is usually detected by URGENCY [18], [19] algorithm. At the moment of crash, the angle of the force or so called "Principal Direction of Force" (PDOF) is calculated in azimuth-elevation coordinate system in the vehicle's body-frame (Fig. 7) by basic trigonometry and noise filtering. The PDOF in the tangent frame depends upon vehicle's attitude at collision.

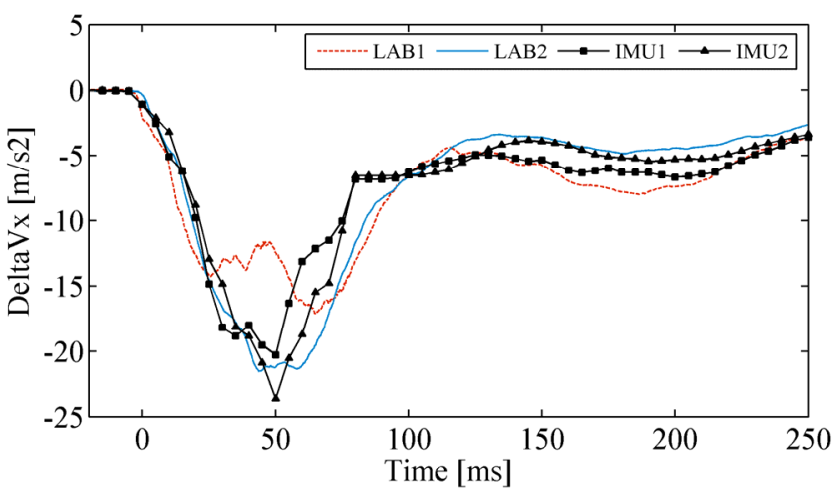

a)

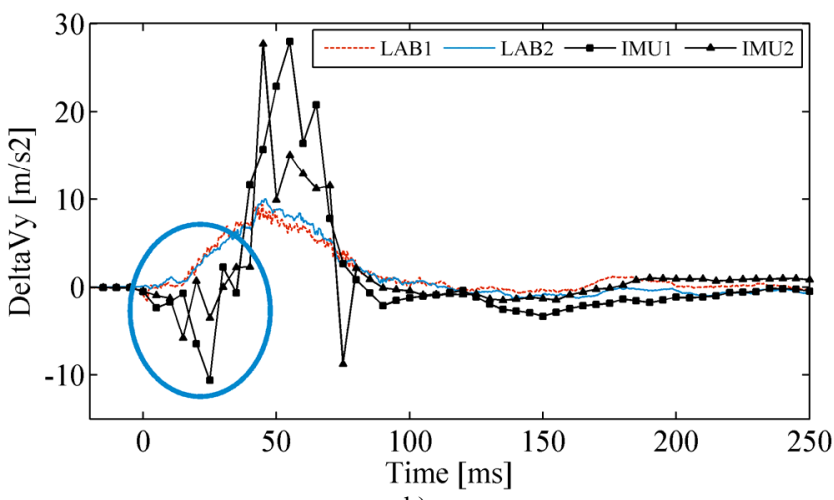

b)

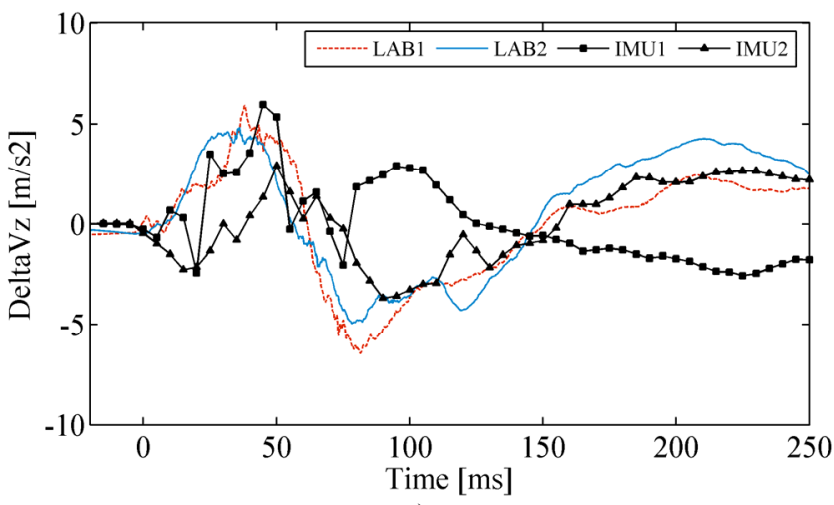

c)

Fig. 8. Rate of change a) longitudinal, b) lateral, c) vertical speed component.
Table 2 lists the PDOF estimates from data captured by four presented platforms (LAB1/2, IMU1/2). The measured vertical PDOFs were very consistent, oppositely to the horizontal ones. The root cause of this discrepancy is found in Fig. 8 which displays the rate of change of speed vector "delta-V". At the moment of crash detection, devices IMU1/2 have recorded a negative peak on the lateral axis (blue circle), which led to a differing estimation of horizontal PDOF. Causes may be different mountings or mounting positions (structural deformation) as IMU1/2 were attached to the vehicle chassis through the floor, whereas LAB1/2 were attached to the B-pillars. These types of imperfections are hard to avoid in practice if IMUs are mounted after the vehicle fabrication.

At impact, as a reaction to the extreme forces, vehicle experienced unconstrained motion. Vehicle position was

\begin{tabular}{|c|c|c|c|c|}
\hline Device & $\begin{array}{l}\text { Horizontal } \\
\text { Angle } \\
\text { (body) }\end{array}$ & $\begin{array}{l}\text { Vertical } \\
\text { Angle } \\
\text { (body) }\end{array}$ & $\begin{array}{l}\text { Horizontal } \\
\text { Angle } \\
\text { (NED) }\end{array}$ & $\begin{array}{l}\text { Vertical } \\
\text { Angle } \\
(N E D)\end{array}$ \\
\hline REF1 & $-10^{\circ}$ & $14^{\circ}$ & \multirow{4}{*}{\multicolumn{2}{|c|}{$\begin{array}{l}\text { In this specific crash" } \\
\text { body" and "NED" frames } \\
\text { were aligned ( } \rightarrow \text { the same } \\
\text { PDOF). }\end{array}$}} \\
\hline REF2 & $-6^{\circ}$ & $11^{\circ}$ & & \\
\hline LCEDR1 & $33^{\circ}$ & $11^{\circ}$ & & \\
\hline LCEDR2 & $43^{\circ}$ & $15^{\circ}$ & & \\
\hline
\end{tabular}

Tab. 2. Measured principal direction of force (PDOF).

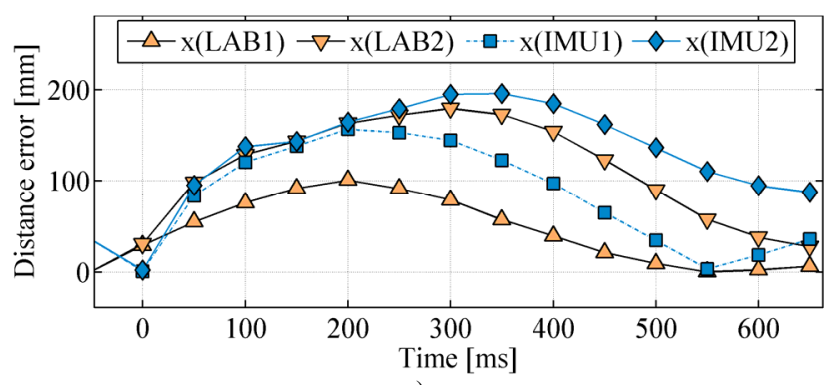

a)

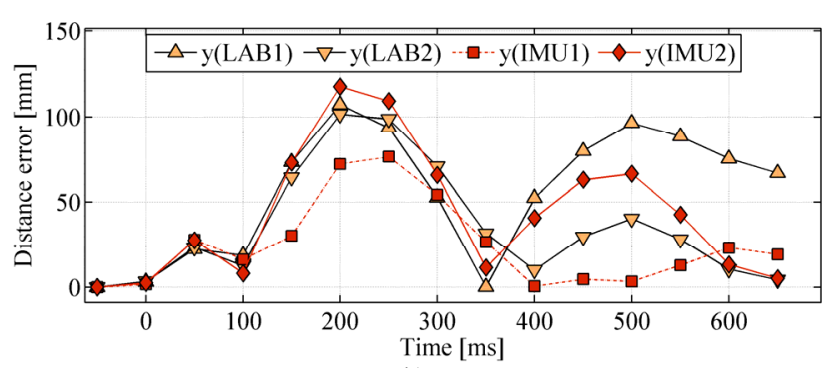

b)

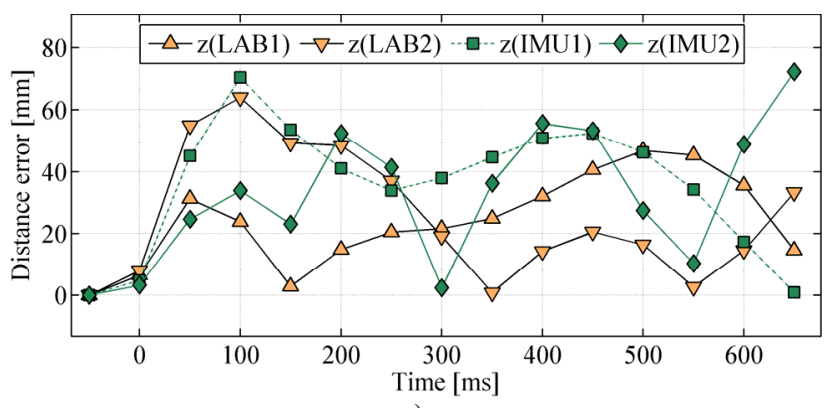

c)

Fig. 9. Measured error of a) longitudinal b) lateral c) vertical displacement of vehicle. 
estimated using SDINS with simplified sensor error model matched to this class of sensors. As crash test labs usually do not provide referent rate gyroscopes, low accuracy component from the IMU1/2 was a single available rate gyroscope in test. Thus, the only available reference position was the one extracted from camera recordings.

Figure 9 shows, respectively, absolute longitudinal, lateral, and the vertical vehicle displacement of LAB1/2, and IMU1/2 in NED frame, relative to camera recording, within the harshest section of the collision of $750 \mathrm{~ms}$. The difference between the trajectory extracted from the video and by the inertial sensor's integration was at the decimeter level in all of the four cases. As testing was limited to a very short time of the initial sensor platform alignment, among causes of errors there are surely imperfect alignment and inaccurate removal of sensor biases and the scale factors.

\section{Conclusion}

The role of inertial and integrated navigation systems for accident analysis and reanalyzing the data in broader terms to promote a better understanding of vehicle and driver behavior before crashes can be enormous.

The experimental results have shown that consumer grade MEMS can become a very interesting source of collision data for expert witnesses and that can be used for short-term reconstruction of vehicle movement in space near crash with acceptable limitations. The approach was validated at an approved safety performance assessment laboratory using custom developed hardware and software. Trajectory extracted from the video and computed using sensor data were within decimeter level on recorded timewindow, which is satisfactory for practical use in vast majority of cases. Using GNSS receiver and coupling algorithm to estimate inertial sensors errors would improve presented results and allow trajectory reconstruction over longer period of time.

\section{References}

[1] GLEAVE, S. D., FRISONI, R., DIONORI, F., et al. Technical development and implementation of event data recording in the road safety policy. Study for European Parliament, September 2014. DOI: $10.2861 / 67672$

[2] GILLIÉRON, P.-Y. From GNSS to black box for challenging road applications. In Toulouse Space Show'12. Toulouse (France), June $25-28,2012$.

[3] SINGH, G. B., SONG, H. Using hidden Markov models in vehicular crash detection. IEEE Transactions on Vehicular Technology, March 2009, vol. 58, no. 3, p. 1119-1128. DOI: 10.1109/TVT.2008.928904

[4] TITTERTON, D. H., WESTON, J. L. Strapdown Inertial Navigation Technology. AIAA, vol. 207, 2004. DOI: 10.1049/PBRA017E

[5] Clausen, P., GILliÉRON, P.-Y., PERAKIS, H., et al. Positioning accuracy of vehicle trajectories for road applications.
In Proceedings of ITS World Congress 2015. Bordeaux (France), October, 5-9, 2015, EPFL-CONF-213568.

[6] JAKUBOV, O., KOVAR, P., KACMARIK, P., et.al. Distributed extended Kalman filter for position, velocity, time estimation in satellite navigation receivers. Radioengineering, 2013, vol. 22, no. 3, p. 776-790. ISSN: 1210-2512

[7] DTS, Solution in Motion, Crash Test Technology Int., UKIP Media \& Events, UK, Sept. 2013, p. 18-19. ISSN: 2051-6096

[8] STEBLER, Y., GUERRIER, S., SKALOUD, J. An approach for observing and modeling errors in MEMS-based inertial sensors under vehicle dynamic. IEEE Transactions on Instrumentation and Measurement, 2015, vol. 64, no. 11, p. 2926-2936. DOI: 10.1109/TIM.2015.2444239

[9] STEBlER, Y., GUERRIER, S., SKAlOUD, J., et al. Study of MEMS-based inertial sensors operating in dynamic conditions. In Proceedings of Position, Location and Navigation Symposium IEEE/ION PLANS 2014. Monterey (CA, USA), May 2014, p. 1227-1231. DOI: 10.1109/PLANS.2014.6851497

[10] BJÖRKHOLM, P., LANDÉN, L., STIGWALL, J., et al. Navigation in vehicle crash test using MEMS-based IMU. In Proceedings of Position, Location and Navigation Symposium IEEE/ION PLANS 2010. Indian Wells (CA, USA), May 2010, p. 27-31. DOI: 10.1109/PLANS.2010.5507134

[11] VERMA, M. K., LANGE, R. C., MCGARRY, D. C. A study of US crash statistics from automated crash notification data. In 20th International Tech. Conference on the Enhanced Safety of Vehicles. Lyon (France), 2007, p. 18-21.

[12] HUANG, M. Vehicle Crash Mechanics. New York: CRC Press, 2002. DOI: $10.1115 / 1.1584416$

[13] SALYCHEV, O. Applied Inertial Navigation Problems and Solutions. Moscow (Russia): BMSTU Press, 2004.

[14] FLENNIKEN IV, W. S., WALL, J. H., BEVLY, D. M. Characterization of various IMU error sources and the effect on navigation performance. In Proceedings of the 18th International Technical Meeting of the Satellite Division of the Institute of Navigation (ION GNSS 2005). Long Beach (CA, USA), September 2005, p. 967-978.

[15] SKOG, I., HÄNDEL, P. In-car positioning and navigation technologies - A survey. IEEE Transactions on Intelligent Transportation Systems, 2009, vol. 10, no. 1, p. 4-21. DOI: 10.1109/TITS.2008.2011712

[16] SOCIETY OF AUTOMOTIVE ENGINEERS. Surface Vehicle Recommended Practice: Instrumentation for Impact Tests - Part 1- Electronic Instrumentation - SAE J211/1 201404, Revised March 2014

[17] INTERNATIONAL STANDARDS ORGANIZATION. Road Vehicles - Measurement Techniques in Impact Tests Instrumentation - ISO 6487:2012, Genève, Switzerland. Revised 2012.

[18] MAlliaris, A., DIGGES, K., DEBlOIS, J. Relationships between crash casualties and crash attributes. SAE Technical Paper 970393, 1997.

[19] AUGEnStein, J., PERDECK, E., STRATTON, J., et al. Characteristics of crashes that increase the risk of serious injuries. In Annual Proceedings of the Association for the Advancement of Automotive Medicine, 2003, vol. 47, p. 561-576.

\section{About the Authors ...}

Srdjan TADIC received his B.Sc. and M.Sc. degrees in Telecommunications from University of Belgrade. He is 
now a Ph.D. candidate in the School of Electrical Engineering, University of Belgrade and VP in company Bitgear. His current research interests include inertial navigation systems, global positioning systems, sensor fusion algorithms and particularly use of GNSS and MEMS sensors in high dynamics environment. He has co-authored 20 journal and conference papers and has 3 patents pending.
Milan B. VUKAJLOVIC received his B.Sc. and M.Sc. degrees in Electronics Department in the School of Electrical Engineering, University of Belgrade. He is now a Ph.D. candidate in the School of Electrical Engineering, University of Belgrade. His current research interests are mainly in the fields of inertial navigation, sensor data fusion and signal processing. 